\title{
Efficacy and safety of meropenem- clavulanate added to linezolid-containing regimens in the treatment of MDR-/XDR-TB
}

\begin{abstract}
Saverio De Lorenzo****, Jan Wilem Alffenaar ${ }^{\#, * *}$, Giovanni Sotgiu“, Rosella Centis ${ }^{+}$, Lia D’Ambrosio ${ }^{+}$, Simon Tiberi*, Mathieu S. Bolhuis ${ }^{\#}$, Richard van Altena ${ }^{\S}$,

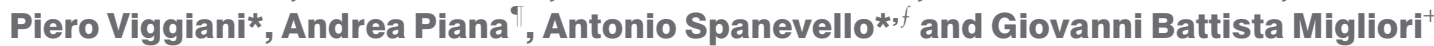

ABSTRACT: Clinical experience on meropenem-clavulanate to treat tuberculosis (TB) is anecdotal (according to case reports on 10 patients). The aim of our case-control study was to evaluate the contribution of meropenem-clavulanate when added to linezolid-containing regimens in terms of efficacy and safety/tolerability in treating multidrug-resistant (MDR) and extensively drug-resistant (XDR) TB cases after 3 months of second-line treatment.

37 cases with MDR-/XDR-TB were prescribed meropenem-clavulanate ( $3 \mathrm{~g}$ daily dose) in addition to a linezolid-containing regimen (dosage range $300-1200 \mathrm{mg} \cdot \mathrm{day}^{-1}$ ), designed according to international guidelines, which was prescribed to 61 controls.

The clinical severity of cases was worse than that of controls (drug susceptibility profile, proportion of sputum-smear positive and of re-treatment cases). The group of cases yielded a higher proportion of sputum-smear converters (28 (87.5\%) out of 32 versus nine $(56.3 \%)$ out of 16 ; $p=0.02)$ and culture converters $(31(83.8 \%)$ out of 37 versus $15(62.5 \%)$ out of $24 ; p=0.06)$. Excluding XDR-TB patients (11 (11.2\%) out of 98 ), cases scored a significantly higher proportion of culture converters than controls $(p=0.03)$. One case had to withdraw from meropenemclavulanate due to increased transaminase levels.

The results of our study provide: 1) preliminary evidence on effectiveness and safety/tolerability of meropenem-clavulanate; 2) reference to design further trials; and 3) a guide to clinicians for its rationale use within salvage/compassionate regimens.

KEYWORDS: Efficacy, linezolid-containing regimens, meropenem-clavulanate, multidrugresistant tuberculosis, safety, tolerability

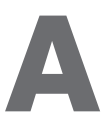
ccording to the World Health Organization (WHO), an estimated 650000 prevalent cases of multidrug-resistant tuberculosis (MDR-TB) occurred globally in 2010 [1-4]. The scale of the problem is alarming, as 12 countries have reported nationwide or sub-national proportions of MDR-TB of $\geqslant 6 \%$ among new tuberculosis (TB) cases and five of these countries also reported MDRTB proportions of $\geqslant 50 \%$ among previously treated cases [1-5]. In Minsk, Belarus, over 50\% of TB cases are presently affected by MDR-TB [6].

WHO estimated 290000 new cases of MDR-TB among notified cases of pulmonary TB in 2010, of whom only around 50000 were reported to have been enrolled on treatment $[1-3,5]$. Overall, $5.4 \%$ of MDR-TB cases were found to be affected by extensively drug-resistant (XDR)-TB strains of Mycobacterium tuberculosis [1-3, 5]. Furthermore, new strains that are resistant to all drugs tested have been described in Italy, Iran and India [7-9], and the international community is still discussing whether the proposed acronym of TDR (totally drug resistant)-TB is adequate to describe the patterns of drug resistance beyond XDR-TB [10].

The difficulty in identifying at least four active drugs, suitable to be included in a multidrug regimen effective against these complicated forms of $\mathrm{TB}$ according to WHO recommendations, makes the need for new antibiotics extremely urgent [11]. Preliminary evidence for three new drugs, presently in the development pipeline (delamanid, bedaquiline and PA-824), has been published recently $[1,12,13]$.

Delamanid, in combination with a background regimen developed according to the WHO guidelines, is associated with an increase in sputumculture conversion at 2 months in patients with
AFFILIATIONS

${ }^{\star}$ AOWV E. Morelli Hospital, Reference Hospital for MDR and HIV-TB, Sondalo,

"Clinical Epidemiology and Medical Statistics Unit, Dept of Biomedical

Sciences, University of Sassari,

Research, Medical Education and

Professional Development Unit, AOU Sassari, Sassari,

${ }^{+}$World Health Organization

Collaborating Centre for Tuberculosis and Lung Diseases, Fondazione S.

Maugeri, Care and Research Institute, Tradate, and

fUniversità degli Studi dell'Insubria, Varese, Italy.

\#Dept of Hospital and Clinical Pharmacy, University of Groningen, University Medical Center Groningen, Groningen, and

${ }^{5}$ Tuberculosis Center Beatrixoord, University of Groningen, University Medical Center Groningen, Haren, The Netherlands.

**Both authors contributed equally.

CORRESPONDENCE

G.B. Migliori Battista, World Health

Organization Collaborating Centre for Tuberculosis and Lung Diseases, Fondazione S. Maugeri, Care and Research Institute, Via Roncaccio 16 , 21049, Tradate, Italy

E-mail: giovannibattista.migliori@ fsm.it

Received:

Aug 082012

Accepted after revision:

Aug 282012

First published online:

Sept 202012

European Respiratory Journal

Print ISSN 0903-1936

Online ISSN 1399-3003 
MDR-TB [11, 12]. A recent multiple-agent combination study assessed that the 14-day early bactericidal activity of PA$824+$ moxifloxacin+pyrazinamide results were significantly higher than those of bedaquiline, bedaquiline+pyrazinamide, PA$824+$ pyrazinamide and bedaquiline+PA-824, and comparable to those of the standard treatment regimen of isoniazid, rifampicin and pyrazinamide with streptomycin or ethambutol [13].

In parallel with further research on the best way to combine these new drugs into new regimens, additional evidence is necessary to confirm the clinical usefulness of existing drugs presently used "off label" to manage difficult-to-treat MDRand XDR-TB cases. Mounting evidence indicates that the efficacy of linezolid is limited by its toxicity [14, 15].

The association of a $\beta$-lactam antibiotic with a $\beta$-lactamase inhibitor has been explored recently with apparently suboptimal results, as $M$. tuberculosis is protected from $\beta$-lactams antibiotics through its potent $\beta$-lactamase encoded by a gene located in the chromosome and called BlaC [16, 17]. Fortunately, clavulanate (a $\beta$-lactamase inhibitor) has demonstrated in vitro the capacity to inhibit the activity of BlaC-coded products [18]. Meropenem, a carbapenem offering a limited substrate to hydrolysis, has demonstrated high bactericidal in vitro activity when combined to clavulanate against susceptible MDR- and XDR-TB strains of M. tuberculosis and the capacity to sterilise cultures in vivo within 2 weeks $[19,20]$.

At present the clinical experience on meropenem-clavulanate is anecdotal and limited to case reports of no more than 10 patients where the drug was prescribed for salvage purposes $[16,19]$. Taking advantage of the existing collaboration and established research protocols, based on WHO guidelines, in TB reference centres in Sondalo, Italy, and in Haren, the Netherlands [11, 15], a study was conducted to evaluate the therapeutic contribution of meropenem-clavulanate when added to linezolid-containing regimens during hospital stay in terms of efficacy, as well as safety and tolerability profile in treating MDR- and XDR-TB cases after 3 months of therapy with second-line drugs.

\section{MATERIAL AND METHODS}

An observational case-control study was carried out in two centres that specialised in the management of complicated TB cases. Adult patients (i.e. aged $\geqslant 15$ years) with cultureconfirmed MDR-TB (i.e. TB caused by M. tuberculosis isolates resistant to at least isoniazid and rifampicin) were consecutively selected and enrolled in both centres.

Resistance to first- and second-line anti-TB drugs was assessed by the quality-assured laboratories located in both centres and confirmed by the WHO Supranational Reference Laboratories of Milan, Italy, and the National Tuberculosis Reference Laboratory, National Institute for Public Health and the Environment, Bilthoven, the Netherlands.

On the basis of the drug-susceptibility testing, anti-TB regimens were administered following the WHO recommendations [11]. Both centres pioneered the off-label use of linezolid between 2001 and 2004. Clinicians working in Sondalo started to prescribe meropenem-clavulanate in 2009.

Cases were individuals treated with an anti-TB meropenemclavulanate-containing regimen, which also included linezolid in all but five patients. The cases were included in the Italian cohort, treated in Sondalo Hospital. The reasons for not prescribing linezolid were the: availability of four effective drugs $(n=3)$; lack of drug-susceptibility testing on linezolid in the presence of four effective alternative drugs $(n=1)$; and concurrent anaemia $(n=1)$.

Controls were treated in Haren Hospital and were subjects whose linezolid-containing regimen did not include meropenem-clavulanate. During hospital admission, meropenemclavulanate was prescribed intravenously at a dose of $1 \mathrm{~g}$ three times a day whereas linezolid was given at a dose ranging from 300 to $1200 \mathrm{mg}$ per day after adjusting the dose based on blood levels. The ratio between cases and controls was 1:2. Drug prescription was not blinded or randomised, following only the drug-susceptibility testing results as per WHO guidelines [11].

Ethical approval for the collection and analysis of anonymous and retrospective data and for the compassionate use of the drugs was obtained by institutional review boards of the participating institutions as per legislation (formal approval not needed in the Netherlands).

Epidemiological, clinical and microbiological information was collected from clinical files on standardised ad hoc electronic forms. In particular, the following covariates were recorded: date of admission; date and place of birth; sex; residence and immigration from a high-burden TB country; HIV positivity; exposure to antiretroviral drugs; previous TB diagnoses; previous anti-TB treatments (i.e. exposure to anti-TB drugs for $>1$ month) and previous treatment outcomes; drugsusceptibility testing results, including susceptibility or resistance to the drugs defining XDR-TB (i.e. TB caused by $M$. tuberculosis strains resistant to isoniazid, rifampicin, any fluoroquinolones and at least one second-line injectable antiTB drug (amikacin, capreomycin, kanamycin)); radiological findings; anti-TB regimen administered (including dosage and length of exposure); surgery; duration of exposure to linezolid and meropenem-clavulanate; adverse events potentially ascribed to the study drugs (i.e. linezolid and meropenem-clavulanate); management of adverse events; proportion of sputum-smear and -culture positivity at hospital admission and at 30,60 and 90 days after the prescription of second-line anti-TB drugs; and time to sputum-smear and -culture conversion.

Qualitative and not normally distributed quantitative variables were statistically evaluated with the Chi-squared test and the Wilcoxon Mann-Whitney tests, respectively. Normality distribution of the continuous data was tested with the ShapiroWilk test. A p-value $>0.05$ was considered not statistically significant. All the statistical calculations were performed using the statistical software Stata 9.0 (StataCorp, College Station, TX, USA).

\section{RESULTS}

98 MDR-TB patients were enrolled in the study: 37 cases and 61 controls were treated with individualised anti-TB meropenem-clavulanate-containing and -sparing regimens (table 1). The former were managed in the Italian reference centre in Sondalo, and the latter in the Dutch reference centre in Haren. 


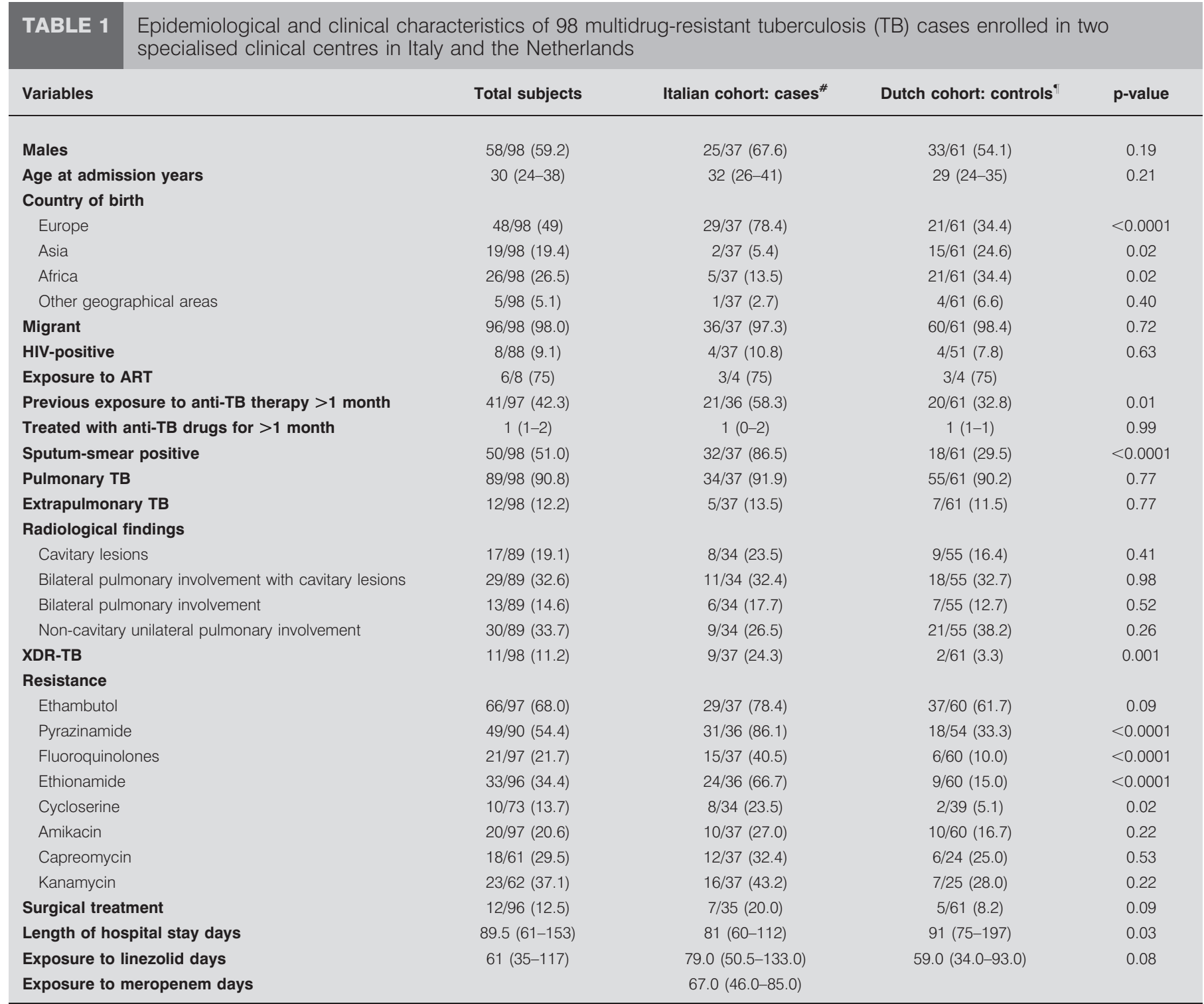

Data are presented as $\mathrm{n} / \mathrm{N}(\%)$ or median (interquartile range), unless otherwise stated. For some entries, the total number of subjects may not be 98 due to missing/ unavailable data. ART: anti-retroviral therapy; XDR: extensively drug-resistant. *: meropenem containing anti-TB regimen; " ${ }^{*}$ meropenem-clavulanate-sparing anti-TB regimen.

Almost $60 \%$ were male, with a median (interquartile range (IQR)) age of $30(24-38)$ years. Most of them were migrants (96 (98.0\%) out of 98), coming from Europe (48 (49\%) out of 98), Africa (26 (26.5\%) out of 98 ) and Asia (19 (19.4\%) out of 98$)$. The percentage of TB/HIV co-infected patients was $<10 \%$ and $75 \%$ of them were treated with antiretroviral drugs.

No statistical differences between cases and controls regarding social and demographic variables were detected, except for the European origin, being significantly higher among cases treated with a meropenem-clavulanate-containing regimen (29 $(78.4 \%)$ out of 37 versus $21(34.4 \%)$ out of $61 ; \mathrm{p}<0.0001)$.

The majority showed pulmonary TB disease (89 (90.8\%) out of 98 ), with only $12 \%$ affected by an extrapulmonary TB. Onethird presented bilateral pulmonary involvement with cavitary lesions, while one-third showed unilateral infiltrates. Half of the patients were sputum-smear positive at hospital admission, with a statistically significant higher prevalence among cases (32 (86.5\%) out of 37 versus $18(29.5 \%)$ out of 61 ; p $<0.0001)$. Overall, almost $13 \%$ of the cases enrolled underwent surgical intervention. The proportion of patients who were prescribed previous anti-TB treatment was higher among the cases treated with meropenem-clavulanate (21 (58.3\%) out of $36)$ than among the controls (20 (32.8\%) out of 61$)$.

Cases treated with a meropenem-clavulanate-containing regimen were infected by $M$. tuberculosis strains showing a worse drug-susceptibility profile, the proportion of XDR-TB cases was significantly higher (nine $(24.3 \%$ ) out of 37 versus two $(3.3 \%)$ out of $61 ; \mathrm{p}=0.001)$, as was the percentage of those harbouring $M$. tuberculosis strains resistant to pyrazinamide 
(31 (86.1\%) out of 36 versus $18(33.3 \%)$ out of $54 ; \mathrm{p}<0.0001)$, fluoroquinolones $(15(40.5 \%)$ out of 37 versus six $(10 \%)$ out of $60 ; p<0.0001)$, ethionamide $(24(66.7 \%)$ out of 36 versus nine $(15 \%)$ out of $69 ; \mathrm{p}<0.0001)$ and cycloserine (eight $(23.5 \%)$ out of 34 versus two $(5.1 \%)$ out of $39 ; \mathrm{p}=0.02)$.

In addition, cases were more likely to be previously treated (21 $(58.3 \%)$ out of 36 versus $20(32.8 \%)$ out of $61 ; p=0.01)$ and to be born in Eastern European countries (28 (75.7\%) out of 37 versus $17(27.9 \%)$ out of $61 ; \mathrm{p}<0.0001)$.

\section{Efficacy analysis}

Patients were treated with a linezolid-containing regimen for a median (IQR) time of 61 (35-117) days. Individuals admitted to the Italian hospital (cases) were exposed to a meropenemclavulanate-containing regimen for a median (IQR) period of 67.0 (46.0-85.0) days. Duration of linezolid and/or meropenem-clavulanate exposure was determined by several parameters, including clinical improvement, sputum-smear and/or -culture conversion and occurrence of life-threatening adverse events. No significant statistical differences were found in the median time to sputum-smear and -culture conversion between cases and controls (46 versus 52.5 days and 46 versus 42 days, respectively). After 90 days of treatment with secondline drugs the proportion of sputum-smear converters was significantly higher in the meropenem-clavulanate-treated patients $(28(87.5 \%)$ out of 32 versus nine $(56.3 \%)$ out of 16 ; $\mathrm{p}=0.02$ ), while the percentage of sputum-culture converters reached a borderline statistical significance (31 (83.8\%) out of 37 versus $15(62.5 \%)$ out of $24 ; \mathrm{p}=0.06)$ (table 2$)$.

Similar results were obtained after the exclusion of the five meropenem-clavulanate-treated patients not exposed to linezolid (table 3).

A sub-analysis, which excluded the XDR-TB patients (11 $(11.2 \%)$ out of 98$)$, to partly correct the worse drug-susceptibility testing pattern of cases when compared to controls, showed that a significantly higher percentage of cases exposed to meropenem-clavulanate achieved sputum-culture conversion $(p=0.03)$ (fig. 1$)$.

\section{Safety and tolerability analysis}

$15(40.5 \%)$ out of 37 cases and seven $(11.5 \%)$ out of 61 controls experienced adverse reactions following drug prescription $(p=0.001)$. The higher proportion of adverse events among cases was almost entirely related to linezolid (12 (37.5\%) out of 32 versus seven (11.5\%) out of 61 ; p-value: 0.003$)$ and thought to be the consequence of exposure to a superior dosage $\left(>600 \mathrm{mg} \cdot \mathrm{day}^{-1}\right)$ of linezolid $(21(65.6 \%)$ out of 32 versus 18 $(29.5 \%)$ out of $61 ; \mathrm{p}=0.001)$.

$13 \%$ (12 out of 93 ) of the selected patients managed in both centres experienced adverse events potentially caused by linezolid which required interruption of the drug (table 4).

Those treated with a daily linezolid dosage of $\leqslant 600 \mathrm{mg}$ interrupted their regimen less frequently due to adverse effects attributable to linezolid when compared with patients exposed to a daily dosage of $>600 \mathrm{mg}$ (three $(5.6 \%)$ out of 54 versus nine $(23.1 \%)$ out of $39 ; p=0.01)$. No statistical differences between patients treated with $<600 \mathrm{mg}$ and $>600 \mathrm{mg}$ were found with regard to anaemia, leukopenia, peripheral neuropathy and gastrointestinal disorders.

Only five (13.5\%) patients out of 37 experienced adverse events potentially attributed to meropenem-clavulanate. In all cases, diarrhoea was present and did not require withdrawal of the drug. In addition, two out of the five cases experienced transient increase of liver function tests. Meropenem-clavulanate was stopped and re-started after 1 week. While in one case meropenem-clavulanate was continued without problems, the other case saw a renewed increase in transaminase levels; the drug was stopped and transaminases normalised rapidly.

\section{DISCUSSION}

The aim of our study was to assess the therapeutic contribution of meropenem-clavulanate when added to linezolid-containing regimens during hospital stay in terms of efficacy, safety and

TABLE 2 Treatment outcomes of 98 multidrug-resistant tuberculosis (TB) cases enrolled in two specialised clinical centres in Italy and the Netherlands after 30,60 and 90 days of treatment

\begin{tabular}{|c|c|c|c|c|}
\hline Variables & Total & Italian cohort: cases ${ }^{\#}$ & Dutch cohort: controls & p-value \\
\hline \multicolumn{5}{|l|}{ Sputum-smear conversion } \\
\hline At 60 days of treatment & $27 / 48(56.3)$ & 20/32 (62.5) & $7 / 16(43.8)$ & 0.22 \\
\hline At 90 days of treatment & $37 / 48(77.1)$ & 28/32 (87.5) & 9/16 (56.3) & 0.02 \\
\hline \multicolumn{5}{|l|}{ Sputum-culture conversion } \\
\hline At 90 days of treatment & 46/61 (75.4) & $31 / 37(83.8)$ & $15 / 24(62.5)$ & 0.06 \\
\hline $\begin{array}{l}\text { Days from start of anti-TB therapy to sputum } \\
\text { smear conversion }\end{array}$ & $51(28.0-75.0)$ & $52.5(38.5-65.0)$ & $46.0(6.0-157.0)$ & 0.85 \\
\hline $\begin{array}{l}\text { Days from start of anti-TB therapy to culture } \\
\text { conversion }\end{array}$ & $42(16.5-82.0)$ & $42.0(28.0-65.0)$ & $46.0(13.0-96.0)$ & 0.96 \\
\hline
\end{tabular}

Data are presented as $\mathrm{n} / \mathrm{N}$ (\%) or median (interquartile range), unless otherwise stated. ${ }^{\#}:$ meropenem-clavulanate containing anti-TB regimen; ${ }^{\bullet}$ meropenemclavulanate-sparing anti-TB regimen. 


\begin{tabular}{|c|c|c|c|}
\hline Variables & Italian cohort: cases" & Dutch cohort: controls & p-value \\
\hline \multicolumn{4}{|l|}{ Sputum-smear conversion } \\
\hline At 30 days of treatment & 5/28 (17.9) & $9 / 18(50.0)$ & 0.02 \\
\hline At 60 days of treatment & $17 / 28(60.7)$ & $7 / 16(43.8)$ & 0.28 \\
\hline \multicolumn{4}{|l|}{ Sputum-culture conversion } \\
\hline At 30 days of treatment & $9 / 32(28.1)$ & $12 / 29(41.4)$ & 0.28 \\
\hline At 60 days of treatment & 20/32 (62.5) & $13 / 25(52.0)$ & 0.43 \\
\hline At 90 days of treatment & 26/32 (81.3) & $15 / 24(62.5)$ & 0.12 \\
\hline $\begin{array}{l}\text { Days from start of anti-TB therapy to sputum smear } \\
\text { conversion }\end{array}$ & $52.5(42.0-65.0)$ & $46.0(6.0-157.0)$ & 0.80 \\
\hline
\end{tabular}

Data are presented as $\mathrm{n} / \mathrm{N}(\%)$ or median (interquartile range), unless otherwise stated. ${ }^{*}:$ meropenem-clavulanate containing anti-TB regimen; ${ }^{\circ}$ meropenemclavulanate-sparing anti-TB regimen.
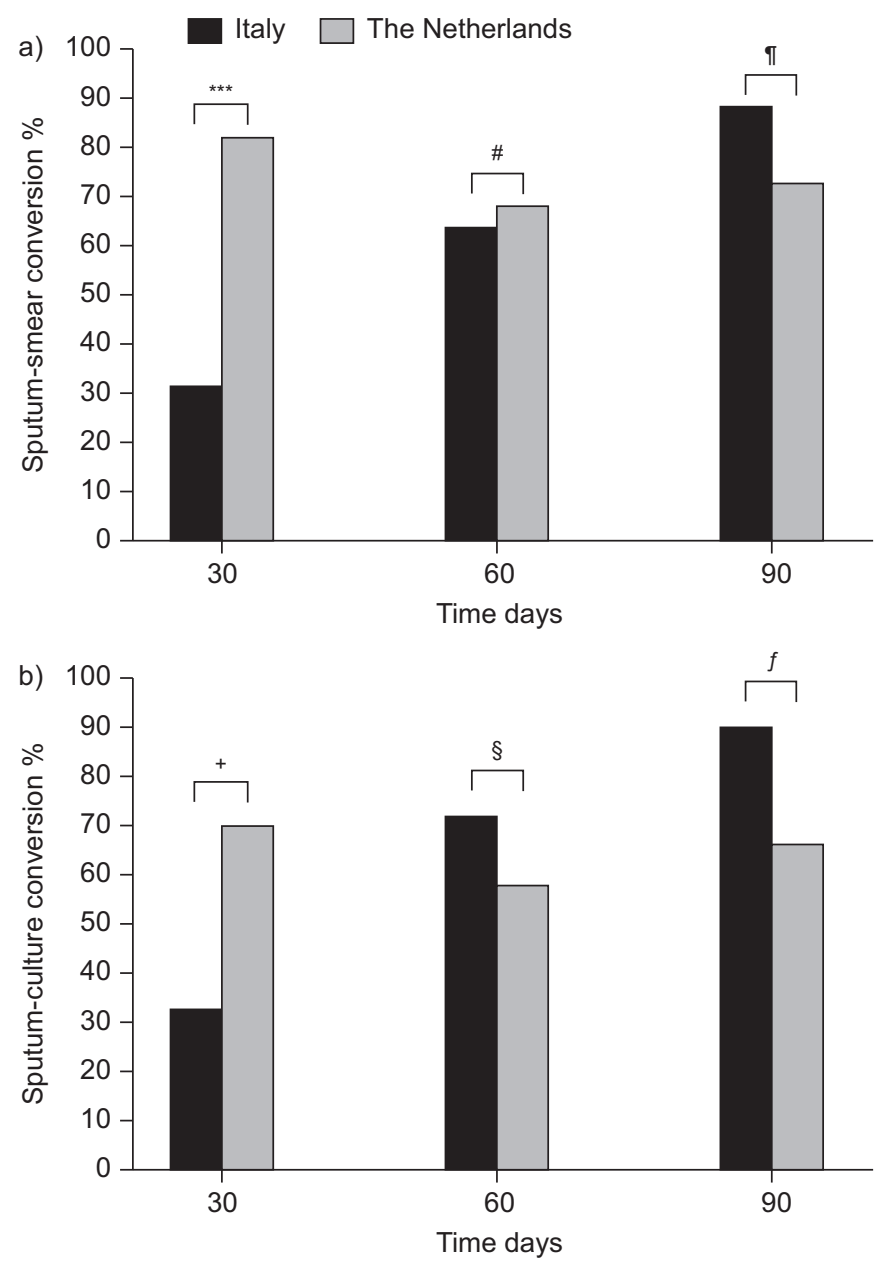

FIGURE 1. a) Sputum-smear conversion and b) culture conversion at 90 days of multidrug-resistant tuberculosis cases, excluding the extensively drug-resistant tuberculosis patients, enrolled in two specialised clinical centres in Italy and the

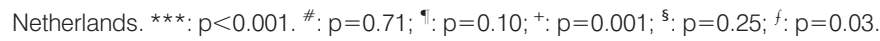

tolerability in treating MDR- and XDR-TB cases after 3 months of treatment with second-line drugs. To our knowledge, this is the first large study evaluating the added value of meropenemclavulanate in managing difficult-to-treat MDR-/XDR-TB cases.

In spite of the worse initial clinical severity of cases (in terms of proportion of re-treatment cases, XDR-TB cases, proportion of sputum-smear positive cases and prevalence of resistance to pyrazinamide, fluoroquinolones, ethionamide and cycloserine), meropenem-clavulanate significantly increased the proportion of sputum-smear conversion in the overall sample, including XDR-TB patients, and sputum-culture conversion among MDRTB cases after 90 days of treatment with second-line drugs.

Furthermore, although the clinical pattern of cases was significantly worse than that of controls (as previously mentioned), the added value of meropenem-clavulanate has already been seen after 60 days of treatment with second-line drugs (with differences not yet significant); although no difference was found in the time to microbiological conversion.

While a comprehensive assessment of culture conversion was performed, unfortunately we could not assess the sputumsmear conversion in all the individuals. This was probably due to a lower pulmonary bacillary load in those with a less severe disease (particularly in the control group) and the effect of previous treatments patients underwent before being admitted to the reference centres.

Importantly, meropenem-clavulanate at an i.v. dosage of $1 \mathrm{~g}$ three times a day was well tolerated and a single episode of drug withdrawal was recorded over a median hospital exposure time of 67 days.

The clinician's decision to interrupt meropenem-clavulanate (a drug only administered intravenously and needing inpatient care) was related to clinical improvement and microbiological conversion (e.g. the conditions allowing hospital discharge) and not to adverse event occurrence in all but one case. 
TABLE 4 Safety and tolerability of linezolid in 93 multidrug-resistant tuberculosis cases

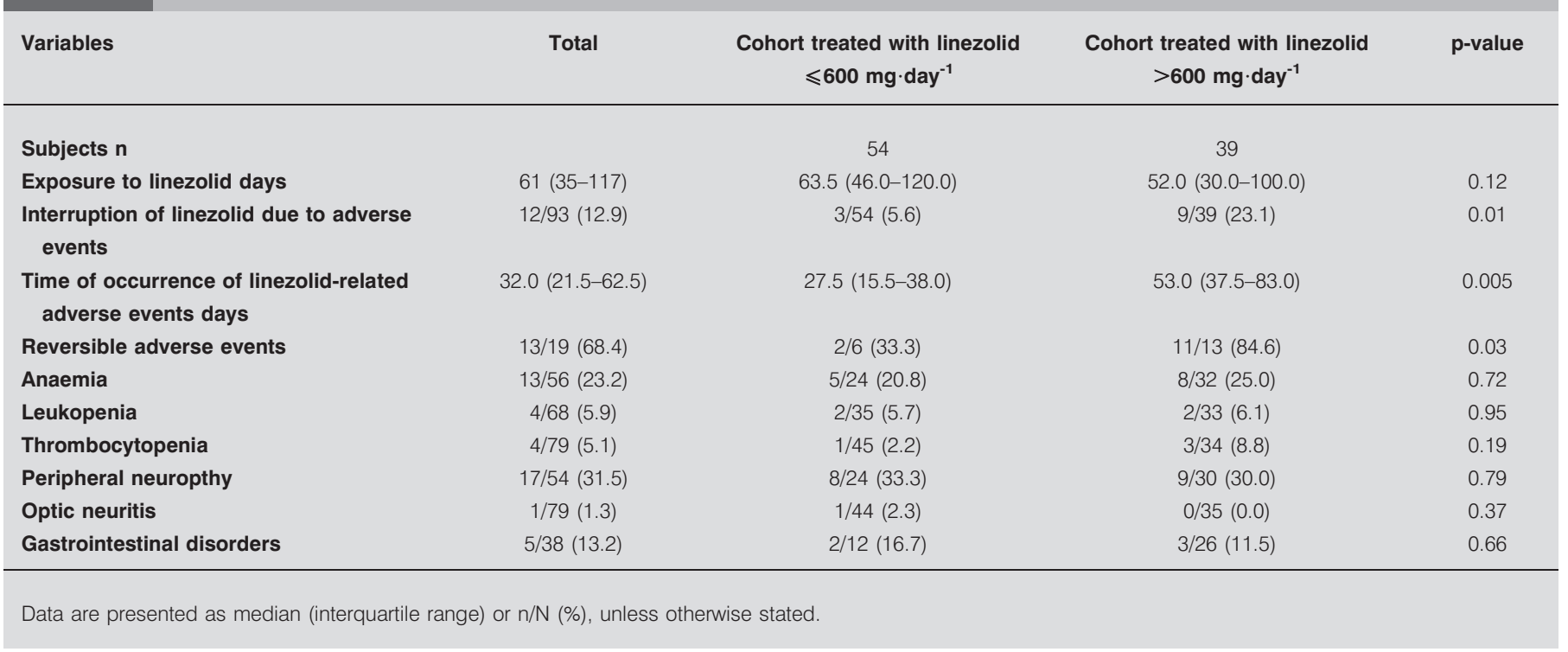

Our study has provided, for the first time, evidence that meropenem-clavulanate increases the proportion of microbiological converters when added to linezolid-containing regimens, designed according to $\mathrm{WHO}$ guidelines [11]. The large sample selected allowed us to obtain more statistical confidence than in previously published case series. In addition, the study was conducted in two specialised centres that had collaborated in the past $[15,21]$ and shared the same protocol to design linezolid-containing regimens and routinely adjust the linezolid dose based on blood levels [15]. The study protocol allowed collection of quality-assured patient information in both centres, making a comprehensive comparison between cases and controls possible.

A further finding of the study is the possibility to add evidence on safety and tolerability of linezolid, taking advantage of 93 cases not included in a recently published individual metaanalysis [15]. While the proportion of major adverse events was lower than previously reported $(12.9 \%)$, the study results confirm the better safety/tolerability of the lower dose ( $\leqslant 600 \mathrm{mg}$ a day) of linezolid [14,15], prescribed more frequently in the Dutch centre and responsible for the significantly lower proportion of total adverse events observed among controls.

Among the main methodological limitations it is worth mentioning the observational, retrospective nature of the study and the consecutive, not randomised enrolment of patients. In addition, although quality controlled by Supranational Reference Laboratories, and distributed among both cases and controls, drugsusceptibility testing for some of the second-line drugs (e.g. cycloserine) needs to be evaluated with caution, given the intrinsic difficulty to perform these tests.

Although the sample size allowed inferential analysis, larger trials will shed further light on the definite role that meropenem-clavulanate might play in treating MDR-/XDRTB cases (e.g. duration of exposure according to clinical severity) when a fourth active drug is needed to design an effective regimen [11].
Although evidence on three new drugs (delamanid, bedaquiline and PA-824) is rapidly accumulating, preceding their market introduction [22], the WHO Group V drugs are still of interest in the management of complicated cases when a fourth active drug is not available either because of the drugsusceptibility testing profile or because of intolerance to active drugs [11]. In addition to continuing research on newly developed drugs and regimens, parallel evidence needs to be raised on existing compounds on which little is proven.

Considering the promising safety/tolerability and efficacy profile of meropenem-clavulanate found in our study, further research on its role and contribution in managing MDR-/XDRTB cases should be encouraged. Furthermore, the 2012 daily cost of meropenem-clavulanate (a 1-g vial three times a day for i.v. use is $\sim € 57$ in Sondalo) is substantially similar to that of linezolid (a 600-mg tablet is $\sim € 55$ and $\sim € 61$ in Sondalo and Haren, respectively) making its use potentially affordable, at least in high-income countries [23, 24].

The results of the present study will provide reference to design trials on meropenem-clavulanate and will guide clinicians for its rationale use within salvage/compassionate regimens $[25,26]$.

\section{STATEMENT OF INTEREST}

None declared.

\section{REFERENCES}

1 Raviglione M, Marais B, Floyd K, et al. Scaling up interventions to achieve global tuberculosis control: progress and new developments. Lancet 2012; 379: 1902-1913.

2 World Health Organization. Global tuberculosis control 2011. WHO/HTM/TB/2011.16. Geneva, WHO, 2011.

3 World Health Organization. Towards universal access to diagnosis and treatment of multidrug-resistant and extensively drugresistant tuberculosis by 2015: WHO progress report 2011. Geneva, WHO, 2011. 
4 Blasi F, Dara M, Van der Werf MJ, et al. Supporting TB clinicians managing difficult cases: the ERS/WHO Consilium. Eur Respir J 2013; 41: 491-494.

5 Zignol M, van Gemert W, Falzon D, et al. Surveillance of antituberculosis drug resistance in the world: an updated analysis, 2007-2010. Bull World Health Organ 2012; 90: 111-119.

6 Skrahina A, Hurevich H, Zalutskaya A, et al. Alarming levels of drug-resistant tuberculosis in Belarus: results of a survey in Minsk. Eur Respir J 2012; 39: 1425-1431.

7 Migliori GB, De Iaco G, Besozzi G, et al. First tuberculosis cases in Italy resistant to all tested drugs. Euro Surveill 2007; 12: E070517.1.

8 Velayati AA, Masjedi MR, Farnia P, et al. Emergence of new forms of totally drug resistant tuberculosis bacilli: super extensively drug-resistant tuberculosis or totally drug resistant strains in Iran. Chest 2009; 136: 420-425.

9 Udwadia ZF, Amale RA, Ajbani KK, et al. Totally drug-resistant tuberculosis in India. Clin Infect Dis 2012; 54: 579-581.

10 Migliori GB, Centis R, D'Ambrosio L, et al. Totally drug-resistant and extremely drug-resistant tuberculosis: the same disease? Clin Infect Dis 2012; 54: 1379-1380.

11 Falzon D, Jaramillo E, Schünemann HJ, et al. WHO guidelines for the programmatic management of drug-resistant tuberculosis: 2011 update. Eur Respir J 2011; 38: 516-528.

12 Gler MT, Skripconoka V, Sanchez-Garavito E, et al. Delamanid for multidrug-resistant pulmonary tuberculosis. N Engl J Med 2012; 366: 2151-2160.

13 Diacon AH, Dawson R, von Groote-Bidlingmaier F, et al. The 14day bactericidal activity of combinations of PA-824, bedaquiline, pyrazinamide and moxifloxacin: the path to novel antituberculosis treatment regimens. Lancet 2012; 380: 986-993.

14 Migliori GB, Eker B, Richardson MD, et al. A retrospective TBNET assessment of linezolid safety, tolerability and efficacy in multidrug-resistant tuberculosis. Eur Respir J 2009; 34: 387-393.

15 Sotgiu G, Centis R, D'Ambrosio L, et al. Efficacy, safety and tolerability of linezolid containing regimens in treating MDR-TB and XDR-TB: systematic review and meta-analysis. Eur Respir J 2012; 40: 1430-1442.

16 Yew WW, Wong CF, Lee J, et al. Do $\beta$-lactam- $\beta$-lactmase inhibitor combinations have a place in the treatment of multidrug-resistant pulmonary tuberculosis? Tubercle Lung Dis 1995; 78: 90-92.

17 Payen MC, De Wit S, Martin C, et al. Clinical use of meropenemclavulanate combination for extensively drug-resistant tuberculosis. Int J Tuberc Lund Dis 2012; 16: 558-560.

18 Hugonnet JE, Blanchard JS. Irreversible inhibition of Mycobacterium tuberculosis $\beta$-lactamase by clavulanate. Biochemistry 2007; 46: 11198-12004.

19 Hugonnet JE, Tremblay LW, Boshoff HI, et al. Meropenem-clavulanate is effective against extensively drug-resistant Mycobacterium tuberculosis. Science 2009; 323: 1215-1218.

20 Payen M, Martin C, Antoine-Moussiaux T, et al. Four cases of XDRtuberculosis treated with meropenem-clavulanate. 50th Interscience Conference on Antimicrobial Agents and Chemotherapy (ICAAC), 2010. L1-517. www.abstractsonline.com/Plan/ViewAbstract.aspx? sKey=7189a738-03a3-41e0-8ee5-cf36269b5766\&cKey=aceae86c-c8ae4d8e-968e-d2dd95abed53\&mKey=\%7b93AEED6A-54D4-4EF6-99BDA9B3CE9FACD9\%7d Date last accessed: April 2, 2013.

21 De Lorenzo S, Centis R, D'Ambrosio L, et al. On linezolid efficacy and tolerability. Eur Respir J 2012; 39: 1-3.

22 Migliori GB, Sotgiu G. Treatment of tuberculosis: have we turned the corner? Lancet 2012; 380: 955-957.

23 Diel R, Rutz S, Castell S, et al. Tuberculosis: cost of illness in Germany. Eur Respir J 2012; 40: 143-151.

24 Loddenkemper R, Sotgiu G, Mitnick CD. Cost of tuberculosis in the era of multidrug resistance: will it become unaffordable? Eur Respir J 2012; 40: 9-11.

25 Raviglione MC, Lange C, Migliori GB. Preventing and managing antimicrobial resistance: an imperative for chest physicians. Eur Respir J 2011; 37: 978-981.

26 Migliori GB, Langendam MW, D'Ambrosio L, et al. Protecting the tuberculosis drug pipeline: stating the case for the rational use of fluoroquinolones. Eur Respir J 2012; 40: 814-822. 